Case Report

\title{
TITLE - MINERAL TRI-OXIDE AGGREGATE (MTA) FOR THE REPAIR OF PERIAPICAL TISSUES IN A PATIENT WITH SELF INFLCTED INJURIES: CASE REPORT
}

\author{
Aditya Shetty', C. Ravi Chandra', Mithra N. Hegde, Uday S. Mahale ${ }^{4} \&$ Ganesh Bhat ${ }^{5}$. \\ ${ }^{1}$ Reader, ${ }^{2}$ Assistant Professor, Sri Sai College of Dental Surgery, Vikarabad, ${ }^{3}$ Senior Professor and Head of Department \\ ${ }^{4}$ Post Graduate Student, ${ }^{5}$ Reader, A.B. Shetty M emorial Institute of Dental Sciences, NItte Univesity, \\ Mangalore - 575018 \\ Correspondence \\ Aditya Shetty \\ Reader, Department of Conservative Dentistry and Endodontics, A.B. Shetty Memorial Institute of Dental Sciences, NItte Univesity, \\ Deralakatte, Mangalore -575018 \\ Mobile : +919886189087 E-mail : shetty_aditya1@yahoo.co.in
}

\begin{abstract}
:
Injury to anterior teeth is a relatively common event. Dentists regularly deal with management of dental trauma and restoration of fractured teeth. Hence the treatment approach and medicaments that assures the biologically acceptable healing and improve long term success rate are of potential value and should be considered.

A big cystic lesion, which is unable to heal non-surgically, heals well with use of M ineral Trioxide Aggregate (MTA Angelus) by surgical approach. Results of clinical trials have recommended the use of M TA asthe most suitable root end filling material.

This article presents the case report of management of big cystic lesion along with the lateral root perforation with maxillary left central incisor. Root canal treatment was followed by the resection of root end of tooth in question at the level of perforation and sealed with MTA. 12 month's follow up radiograph showed completely healed Cystic lesion.
\end{abstract}

Keywords : root canal therapy, apiceoctomy, periapical cyst, root perforation

\section{Introduction:}

Contemporary Endodontic surgical procedures have become major considerations in the management of teeth and associated peri radicular diseases through a biologic rationale and understanding. Today, while non - surgical management is considered as the primary mode of case management, the decision to intervene surgically is often open to deliberation and debate. Endodontic Surgery is thus primarily indicated for three reasons:

If there is a strong possibility of failure of Endodontic therapy alone with non - surgical approaches, surgery is

\begin{tabular}{|c|}
\hline Access this article online \\
\hline Quick Response Code \\
\hline
\end{tabular}
indicated.

If failure has resulted from non - surgical retreatment and retreatment further is not possible or the outcome warrants a surgical approach for better results.
If a biopsy is necessary at or near apex of the tooth, surgery is indicated.

The present Case report focuses on the importance of surgical endodontic intervention and successful restoration of tooth to normal function and form traumatized by self inflicted injury.

\section{Case Report :}

A 24 year old male patient visited the OPD of Department of Conservative Dentistry and Endodontics, A.B. Shetty Memorial Institute of Dental Sciences, Deralakatte, $M$ angalore with the chief complaint of broken teeth in the upper front region of jaw with a swelling on the palate. Two years ago Patient had a fall and he noticed broken front teeth. He reported to local dentist for which an access opening and pulp extirpation was done. Patient did not undergo further dental treatment. The temporary restoration given to close the access opening was fractured. After taking thorough case history, it was revealed that, there was accumulation of food particles in 
opened root canal space, which initiated patient to use safety pins for removal of debris. M edical History was noncontributory and intra oral examination revealed a soft, fluctuant, non - tender swelling in the anterior region of palate with approximate size of $2 \mathrm{~mm} \times 3 \mathrm{~mm}$. Hard tissue examination revealed Ellis Class III fracture with respect to Maxillary Left Central Incisor. Radiographic examination revealed a traumatic injury to maxillary left central incisor in the form of lateral root perforation and periapical loss of lamina dura with a well circumscribed lesion.(Fig 1) A tentative diagnosis of Periapical Cyst was made with respect to Maxillary Left Central Incisor, Maxillary right Central Incisor, M axillary Left Lateral Incisor.

\section{Treatment Plan :}

1. Pulp Space Therapy - Maxillary Left Central Incisor, Maxillary right Central Incisor, Maxillary Left Lateral Incisor.

2. Endodontic Periapical Surgery for enucleation of cyst and repair of lateral root perforation with maxillary left central incisor.

3. Restoration of teeth with Porcelain Fused to Metal crown.

Routine Access Cavities were prepared for maxillary right central incisor and left lateral incisor. Working Length was estimated for all three teeth followed by thorough canal debridement and pulp space preparation (Fig 2,4). Obturation was done by Lateral Condensation method for Maxillary Right Central and Left Lateral incisor (Fig 3). Multiple Calcium hydroxide dressings were given for M axillary Left Central Incisor which were not of any help in healing of periapical radiolucency. Therefore surgery was planned and it was obturated using Rolled Cone Technique and Gutta Flow (Fig5).

Endodontic Periapical Surgery was performed by raising a Leubke - Oschenbein Flap (Fig 6). Cyst Enucleation was done. Apicoectomy with respect to M axillary Left Central Incisor was done to the level of lateral root perforation and M TA (Angelus Indústries, Londrina, Brazil) was placed as Retrograde Restorative Material (Fig 7). Biopsy of enucleated cystic lesion was sent for histopathology report. Silk Sutures were placed in surgical site and patient was recalled periodically for suture removal followed by a one year follow up at intervals of one month, three months, six months and one year (Fig 8). Biopsy report confirmed the diagnosis of Periapical Cyst. 12months follow up showed good bone healing and tooth was asymptomatic (Fig 8).

\section{Discussion :}

Success rates of endodontic initial treatment have been improving over the years, persistence of periapical disease is far from being a rare condition. ${ }^{1}$. The principal modalities available to manage endodontic treatment failures are orthograde retreatment and apical surgery. Both modalities have specific advantages, clinical implications, and risks, and their selection involves a complex decisionmaking process ${ }^{2}$. The most common therapeutic options for the treatment of teeth with periapical pathosis are nonsurgical orthograde treatment and surgical treatment. Selection between alternative treatments should be based on assessment of respective benefits (mainly healing) and risks from studies consistent with a high level of evidence ${ }^{3}$. Current, relevant knowledge on the outcome of endodontic therapy is the key to clinical decision making, particularly when endodontic treatment is weighed against tooth extraction and replacement. Inherent to reviewing the outcome is a definition of "success" in relation to the goals of therapy. As the specific goal set out by the individual patient may either be healing/prevention of disease (apical periodontitis) or just functional retention of the tooth, the potential for both healing and functionality is reviewed. The chance for the teeth to be functional over time is 86 percent to 92 percent. Considering the favorable outcome, conservative endodontic therapy, both nonsurgical and surgical, is definitely justified and should be attempted when a good restorative and periodontal prognosis is projected, unless the patient is not motivated to retain the tooth ${ }^{4}$.

In this case, decision of surgery became mandatory due to inability of repetitive orthograde Calcium hydroxide dressings to heal the periapical tissues and lateral root perforation. 


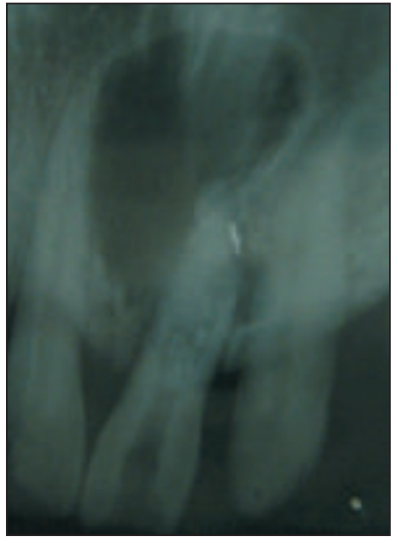

Fig 1 :

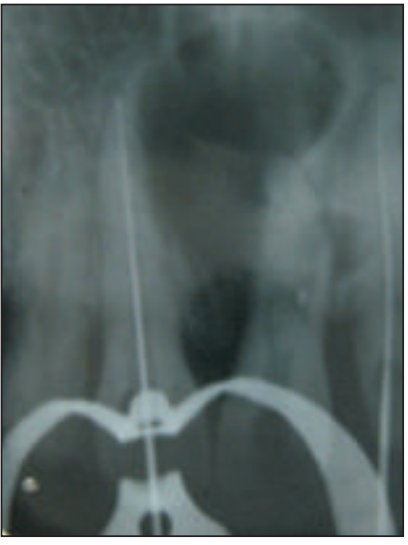

Fig 2 :

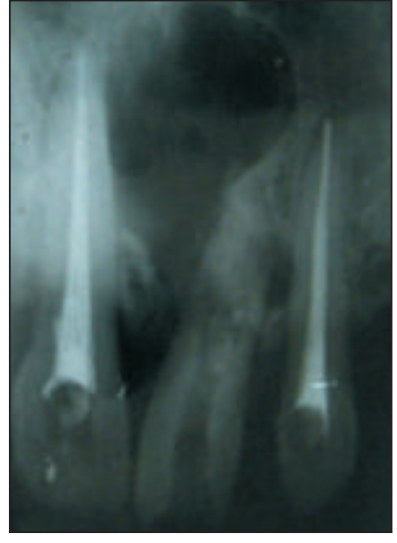

Fig 3 :

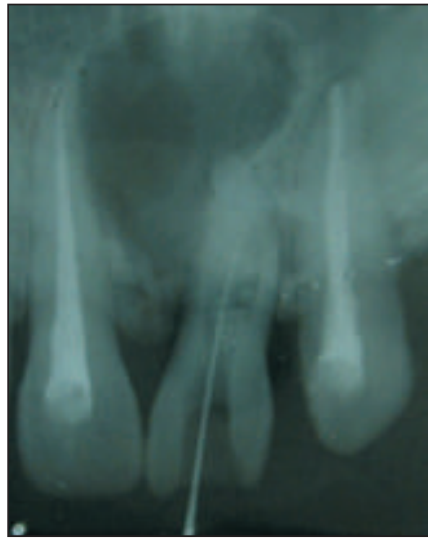

Fig 4 :

Pre - Operative Radiograph Working Length Estimated wrt 11,22 Obturation done w.r.t. 11,22 Working Length Estimated w.r.t. 21

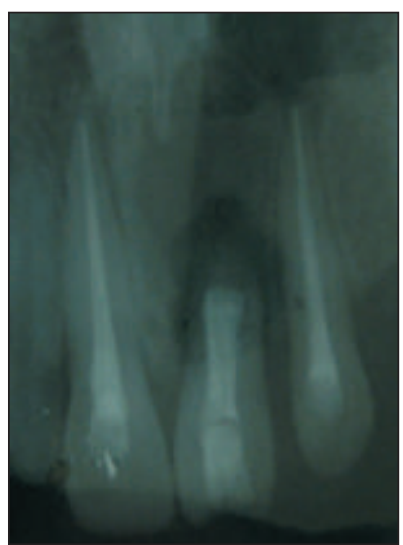

Fig 5 :

Obturation done w.r.t. 21 Using rolled cone technique and Gutta Flow.

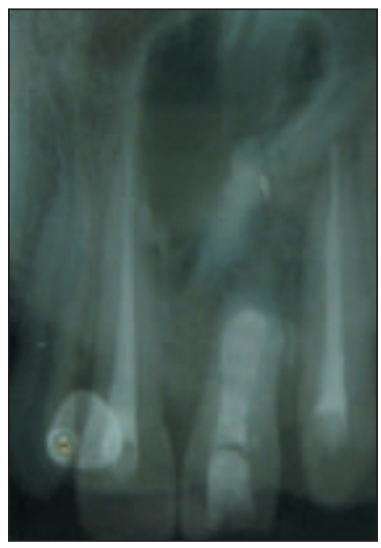

Fig 8 : Three months Post Operative Shows bone healing. Well obturated $11,21,22$ and $M$ TA as retrograde filling material with respect to 21 .
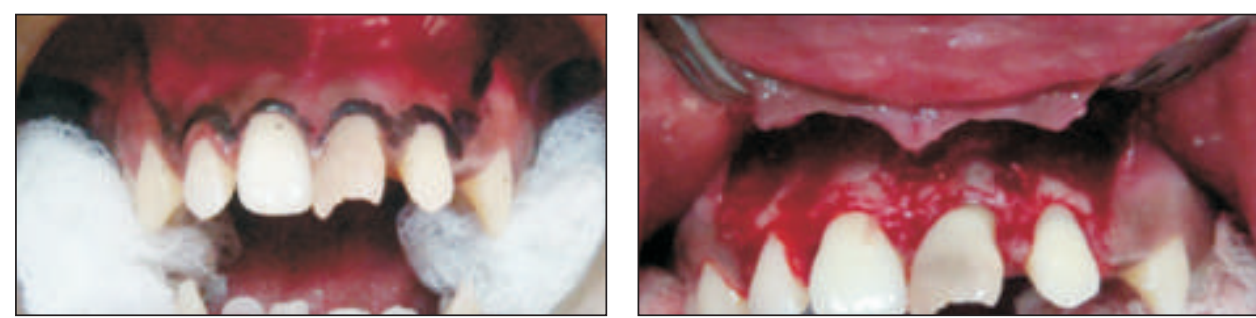

Fig 6 : Surgical Flap - Leubke Oschenbein Flap M arking done and raised.
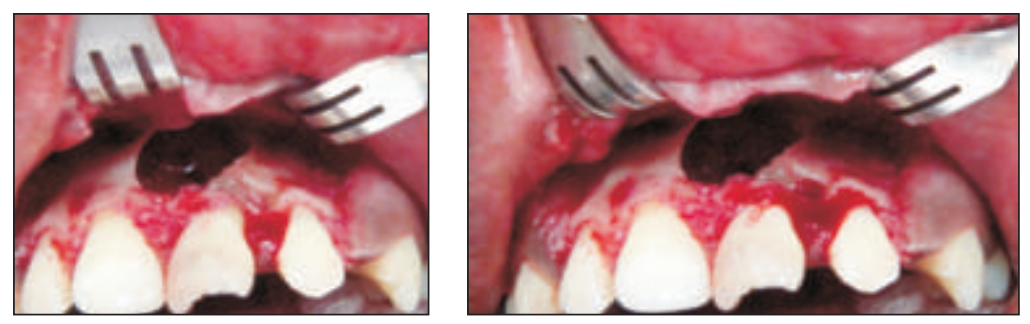

Fig 7 : A Bony window was prepared using slow speed straight handpiece and surgical round bur.

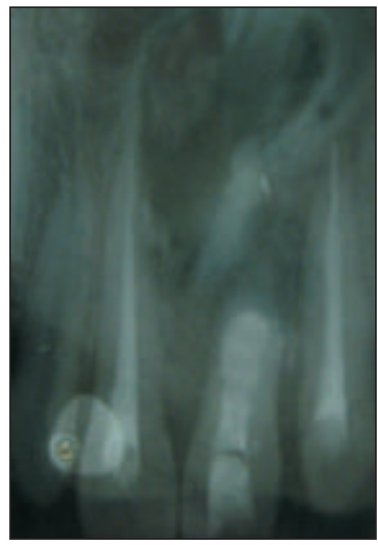

Fig 9 : $12 \mathrm{M}$ onths Post Operative Radiograph shows evidence of good periapical bone healing.
Results of clinical trials have recommended the use of MTA as the most suitable root end filling material. ${ }^{6}$ Results of our treatment supported the fact that, Mineral Trioxide Aggregate (MTA) materials demonstrate biocompatible behavior and acceptable in vivo biologic performance when used for root-end fillings and perforation repairs. ${ }^{7}$

\section{Conclusion :}

From the present case report it is concluded that combined approach i.e. routine pulp space therapy followed by surgical intervention for management of Endodontic periapical lesions of chronicity would positively affect the treatment outcome without requirement of a revision surgery or bone grafts to treat these large osseous defects. 


\section{References:}

1. Del Fabbro M, Taschieri S, Testori T, Francetti L, Weinstein RL. Surgical versus non-surgical endodontic re-treatment for periradicular lesions. Cochrane Database Syst Rev. 2007.18;(3):CD005511.

2. Chalfin $H$, Kellert $M$, Weseley P. Postsurgical endodontics. Journal of Endodontics. 1993;19(6):307-11.

3. Hepworth MJ, Friedman S. Treatment outcome of surgical and nonsurgical management of endodontic failures. Journal of Canadian Dental Association. 1997;63(5):364-71.

4. Friedman S, Mor C. The success of endodontic therapy--healing and functionality. Journal of California Dental Association. 2004;32(6):493-503.

5. Chinni S, Datta K, Srinivasan MR, Kumar ST. Biphasic calcium phosphate in periapical surgery. Journal of Conservative Dentistry 2008;11(2): 92 - 96

6. Von Arx T, Hänni S, Jensen SS. Clinical results with two different methods of root-end preparation and filling in apical surgery: mineral trioxide aggregate and adhesive resin composite. Journal of Endodontics 2010;36(7):1122-9

7. Robertsa H.W., Tothb J.M., Berzinsc D.W., Charlton D.G. Mineral trioxide aggregate material use in endodontic treatment: A review of the literature. Dental M aterials.2008;24:149-164 